\title{
Diagnosis and management of brain metastases: an updated review from a radiation oncology perspective
}

\author{
Gutiérrez-Valencia Enrique, Sánchez-Rodríguez Irving¹, Balderrama-Ibarra Ricardo1, Fuentes-Lara \\ Jesús ${ }^{1}$, Rios-Martínez Alan¹, Vázquez Aldana Arroyo Iñigo', Bayardo-López Luis', Hernández Chávez \\ Allan', Puebla-Mora Ana Graciela², Nader-Roa Liliana ${ }^{3}$, Espíritu-Rodríguez Roque ${ }^{3}$
}

\begin{abstract}
IInstituto Mexicano del Seguro Social (IMSS), Departamento de Radio-Oncología, Centro Médico Nacional de Occidente, Universidad de Guadalajara, Guadalajara 44340, México.

${ }^{2}$ Instituto Mexicano del Seguro Social (IMSS), Departamento de Anatomía Patológica, Centro Médico Nacional de Occidente, Universidad de Guadalajara, Guadalajara 44340, México.

${ }^{3}$ Instituto Mexicano del Seguro Social (IMSS), Departamento de Física Médica, Centro Médico Nacional de Occidente, Guadalajara 44340, México.
\end{abstract}

Correspondence to: Dr. Gutiérrez-Valencia Enrique, Departamento de Radio-Oncología, Centro Médico Nacional de Occidente, Universidad de Guadalajara, Belisario Domínguez 1000, Col. Independencia Oriente, PC 44340, Guadalajara, Jalisco, México. E-mail: jeguval@hotmail.com

\begin{abstract}
How to cite this article: Enrique GV, Irving SR, Ricardo BI, Jesús FL, Alan RM, Iñigo VAA, Luis BL, Allan HC, Graciela PMA, Liliana NR, Roque ER. Diagnosis and management of brain metastases: an updated review from a radiation oncology perspective.

J Cancer Metastasis Treat 2019;5:54. http://dx.doi.org/10.20517/2394-4722.2019.20
\end{abstract}

Received: 6 Mar 2019 First Decision: 12 Apr 2019 Revised: 14 May 2019 Accepted: 16 May 2019 Published: 11 Jul 2019

Science Editor: William Schiemann Copy Editor: Cai-Hong Wang Production Editor: Jing Yu

\begin{abstract}
Brain metastasis are the most common intracranial malignancy in the adult population. Their incidence has increased dramatically over the last 20 years, as a result of the increasing number of cases stemming from lung and breast cancer together with the higher cancer survival rates due to diagnostic and therapeutic advances. More than $40 \%$ of cancer patients develop brain metastases during the course of their disease: specifically, they appear in 50\% of patients with lung cancer, more than $25 \%$ of patients with breast cancer, and $20 \%$ of patients with melanoma. Diagnosis is made using different imaging approaches, such as computed tomography and magnetic resonance imaging, accompanied by clinical manifestations and a history of malignancy supporting the diagnosis of a brain metastasis. Current treatment options should be oriented to the patient's current performance, the number of intracranial and extracranial lesions, and related factors. Although surgical resection and whole-brain radiotherapy have been standard treatments for many years, numerous treatment modalities have become more easily available and accepted worldwide, producing more favorable and reliable results. Among these is stereotactic radiosurgery, and the latest clinical trials support this treatment.
\end{abstract}


Keywords: Brain metastases, whole-brain radiation therapy, stereotactic radiosurgery, graded prognostic assessment

\section{INTRODUCTION}

Brain metastases are the most common intracranial malign neoplasms in adult patients, with 170,000 new cases per year reported in the USA alone ${ }^{[1]}$. The incidence of brain metastasis has been on the rise the last 20 years, resulting from an increase in the number of cases of lung and breast cancer $^{[2]}$ and the fact that cancer survival rates have been increasing thanks to new therapeutic advances and the availability of central nervous system imaging technologies for diagnosis. More than $40 \%$ of patients with cancer develop brain metastases: specifically, they appear in $50 \%$ of patients with lung cancer, more than $25 \%$ of patients with breast cancer, and $20 \%$ of patients with melanoma ${ }^{[3,4]}$.

Multiple epidemiological studies have been conducted regarding brain metastases. These have had different methods and limitations; however, a comparison between them can bring an understanding of the incidence of brain metastasis. Such studies include the initial work done by Goumundsson in Iceland, which reported an incidence of 2.8 cases per 100,000 people ${ }^{[5]}$, and Percy et al. ${ }^{[6]}$, where the incidence found was as high as 11.1 cases per 100,000 . The Barnholtz-Sloan et al. ${ }^{[7]}$ 's study derived greater validity from its use of the register of the Metropolitan Detroit Cancer Surveillance System from 1973 to 2001, carrying information on an approximate population of 4.5 million patients, in which the observed incidence of brain metastasis in patients with any kind of neoplasm was 9.6\%.

Considerable variability in the incidence of brain metastasis has been found, which may be attributable to limited available data, such as autopsy reports or general hospital records; nevertheless, beginning with the first records of cancer, a similar incidence of brain metastasis has been observed, which may be attributable to the fact that it is an exclusively oncological population limited to a particular state or region and follow-up of the same patients ${ }^{[8,9]}$.

Generally, lung cancer is the foremost cause of brain metastasis, with studies reporting incidences of $12 \%$ to $65 \%{ }^{[10]}$ of all patients with primary lung cancer. Among the most commonly associated histologies for brain metastasis are small-cell lung cancer and adenocarcinoma. In a high percentage of patients with lung cancer, diagnosis is performed after the onset of neurological symptoms.

Breast cancer is the main cause of brain metastases in women, with reported incidences between $5 \%$ and $30 \%$ of all breast cancer cases. Unlike the case of lung cancer, in breast cancer the diagnosis of brain metastasis usually follows well after the initial diagnosis of cancer. Diagnoses of melanoma have increased over the last several years, and this malignant neoplasm has the greatest capacity to develop into brain metastasis, with incidences from $12 \%$ to $90 \%$ [Figures 1-3]. Incidences of $7 \%$ to $10 \%$ of all patients with renal cancer and $1 \%$ to $4 \%$ of all gastrointestinal tumors have been reported ${ }^{[11]}$.

The therapeutic management of patients with brain metastases depends on the localization and number of brain lesions, primary tumor biology, and disease extension. The overall survival from the moment of diagnosis for untreated patients is 1-2 months, which can be extended to 6 months in patients who receive conventional radiotherapy and chemotherapy ${ }^{[4]}$.

\section{PATHOPHYSIOLOGY}

The genesis of metastasis requires several complex and sophisticated steps to occur first. These include genetic, epigenetic, and biological changes known as the "metastatic cascade" ${ }^{\text {"[12,13] }}$. This process begins with the detachment of a tumor cell from its primary lesion and the invasion of the surrounding tissue, 




Figure 1. Left parietal lesion

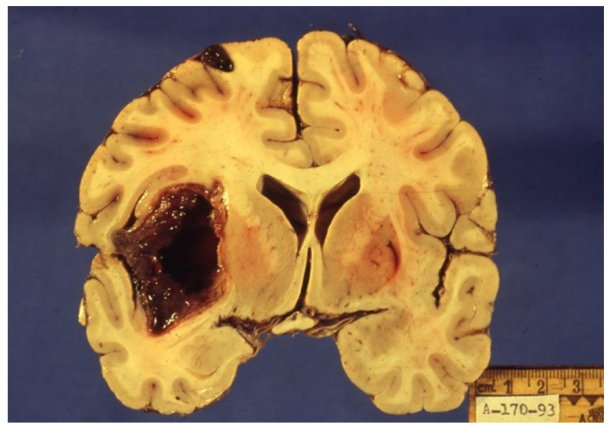

Figure 2. Macroscopic view of a lesion located in the left temporal lobe, with dimensions of $5 \mathrm{~cm} \times 4 \mathrm{~cm} \times 3 \mathrm{~cm}$, from the cystic-necrotic center. A subepidural lesion appears in the parietal region on the same side



Figure 3. Histologically, this lesion is composed of epithelioid-like cells with moderate eosinophilic cytoplasm and well-defined cytoplasmic junctions between some cells. The nuclei are vesicles with an apparent nucleolus. A brown pigment characteristic of melanocytic cells is seen

including the basement membrane, which is followed by intravasation in the blood vessels, hematogenous and lymphatic dissemination, the production of circulating tumor cells in brain capillaries, and then extravasation. Finally, the cancer cells must colonize the surrounding tissue and induce angiogenesis and cell proliferation, forming secondary lesions ${ }^{[14]}$.

The blood-brain barrier is a functional and anatomical barrier that plays an important role in the interaction between the cerebral microenvironment and metastatic colonization ${ }^{[15]}$. In this process, tumor cells survive 
an inflamed cerebral microenvironment that is appropriate for their development and growth, which is known as their niche ${ }^{[16]}$.

Tumor cells can evade growth-suppressing factors and inhibitors of cell proliferation through mechanisms that include resistance to apoptosis, overexpression of Bcl-2 and Bcl-xL, and the inhibition of proapoptotic pathways Bax and $\mathrm{Bim}^{[12]}$. Tumor cells can be dedifferentiated, migrate to a distant site, survive apoptosis, disseminate, and redifferentiate. The activation of cells in the adjacent stroma by paracrine signaling with pro-tumor factors maintains the growth of the tumor, intensifying genomic instability and epigenetic dysregulation ${ }^{[11,13]}$.

Tumor cells adhere to the endothelium of recipient tissue and act as macrophages, creating pseudopods and penetrating cell-to-cell junctions, subsequently gaining access to normal tissue parenchyma to activate angiogenesis and develop new vessels for its nutrition, in this way promoting the growth of secondary injuries ${ }^{[17]}$. Circulating cells attract platelets due to the proteins they express on their surface, which protects them from the immune system. Likewise, metastatic cells activate mechanisms to escape immunity by reducing the expression of TAP 1 , which decreases the effects of T-cell-mediated death ${ }^{[18]}$.

\section{DIAGNOSIS}

Although clinical manifestations of a history of malignant disease with central nervous system metastasis potential may obviously occur, it will not always be possible to obtain histological confirmation of these lesions [Figure 3]. In such cases, complementary image studies may play an important role in determining the best management for these patients.

\section{Image studies}

Magnetic resonance imaging (MRI) is the tool of choice when brain metastasis is suspected, due to its high sensitivity and specificity, which support its high capacity to detect smaller lesions than those that appear in computed tomography (CT) with or without contrast; it is also associated with fewer bone artifacts in posterior fossa. However, if MRI cannot be used, CT is still a valid option ${ }^{[19]}$.

CT will show isodense lesions in contrast with brain parenchyma, which will intensify with the application of intravenous contrast medium. When lesions appear hyperdense, one may suspect secondary bleeding, especially in histologies associated with high spontaneous bleeding risk (including choriocarcinoma, melanoma, and renal carcinoma); there are also other findings that are secondary to lesions and that can be easily visualized, such as hydrocephaly, ring-enhancing cerebral lesions, and brain herniation ${ }^{[19,20]}$.

The sensitivity and specificity of CT scans are $92 \%$ and $99 \%$, respectively, and they are considerably higher in tumors that have a high incidence of central nervous system metastasis, such as non-small-cell lung cancer $^{[21]}$. MRI exhibits an ability to detect lesions smaller than $1 \mathrm{~cm}$, up to $70 \%$ more sensitive than $\mathrm{CT}$, and this increases in cases of multiple metastases ${ }^{[19]}$. MRI has other beneficial characteristics, for example, in the use of distinct sequences such as T1, T2, FLAIR, diffusion, and perfusion, which can be used along with spectroscopy to increase sensibility and specificity ${ }^{[2]}$.

The majority of metastatic lesions are hypointense in T1 sequence images, which can be an indication of hemorrhage and necrosis. In T2 weighted images, the majority of lesions appear hyperintense [Figure 4]. Bleeding appears as acutely hypointense and becomes hyperintense as chronicity develops, while vasogenic edema appears hyperintense. The use of contrast significantly increases its sensitivity and specificity for the detection of brain metastases relative to simple $\mathrm{MRI}^{[23]}$. 


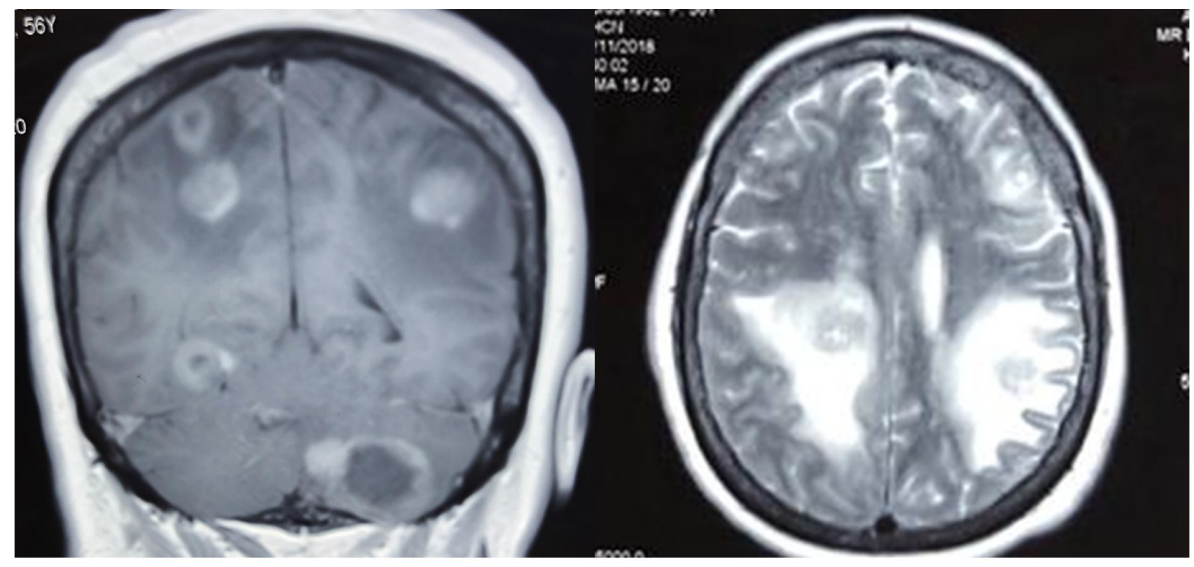

Figure 4. Magnetic resonance imaging of a patient with multiple brain metastases. Axial T2 slice sequence shows perilesional edema in the junction of white and gray matter

Table 1. Spectroscopy

\begin{tabular}{|c|c|c|c|}
\hline Metabolite/marker & Function & As found in brain metastases & Range (parts per million) \\
\hline Creatine & Metabolism & Internal standard & 3.0 \\
\hline Choline & Cellular membrane turnover & Increased & 3.2 \\
\hline Lipids & Necrosis & Increased & $0.9-1.4$ \\
\hline Lactate & Anaerobic metabolism/necrosis & Increased & 1.3 \\
\hline $\mathrm{N}$-acetylaspartate & Neuronal viability & Decreased & 2.0 \\
\hline
\end{tabular}

\section{SPECTROSCOPY}

Spectroscopy is an MRI technique that allows the metabolic characteristics of lesions to be evaluated, which may help distinguish between benign lesions and malignant lesions which would be difficult to differentiate using MRI alone. Spectroscopy can be performed for single or multiple tumor regions (unique voxel or multivoxel) to detect certain ranges of specific metabolites in brain tissue, such as choline, creatinine, lipids, lactate, and $\mathrm{N}$-acetyl-aspartate (NAA) ${ }^{[22,23]}$.

The analysis of these metabolites is helpful for distinguishing metastasis from necrosis, gliosis, and vasogenic edema [Table 1].

Creatine is the most stable metabolite in brain tissue, although it can be diminished in malignant primary tumors such as high-grade gliomas. It is present in both white and gray matter, which enables it to be used as an internal reference for the remainder of metabolites, which can change in the context of metastatic brain disease $^{[24,25]}$.

Choline is a marker of cell change: it is a structural part of cell membrane phospholipids, with a greater presence in white matter than in gray matter. It is elevated where there are high-grade cell changes, and it has a relationship with creatine, such that both appear elevated, which helps orient a diagnosis of brain metastasis ${ }^{[26]}$.

Because lipids are a structural component of cell membranes, they appear elevated in the case of severe cell damage, even with necrosis. Lactate is a main metabolite associated with anaerobic metabolism, and necrosis is common in brain metastases ${ }^{[26]}$. NAA is found at high concentrations in normal brain tissue, making it a marker for cell integrity and normal tissue structure; this marker appears at low concentrations in brain metastases ${ }^{[25,26]}$. 
Spectroscopy may increase the accuracy of MRI in differentiating tumors from necrosis in patients with metastases who have been irradiated, using choline/NAA and choline/creatine ratios ${ }^{[26]}$. According to a meta-analysis of 261 patients, it can also guide a clinician's therapeutic approach by adding information that could contribute to a differential diagnosis of primary malignant tumors such as high-grade gliomas; the study concluded that the most useful spectroscopy parameter is the choline-NAA ratio, preferably using a multi-voxel technique in the peritumoral region ${ }^{[27]}$. However, in contrast, a meta-analysis of advanced MRI metrics that included 24 spectroscopy studies found no reliability of this technology in distinguishing highgrade gliomas from metastases using the choline-creatine ratio; the authors had excluded cases in which the choline-NAA ratio was used due to insufficient data ${ }^{[28]}$.

Hence, there is a lack of robust data supporting the routine use of MRI spectroscopy for differentiating metastases from high-grade gliomas, but it remains an important tool supporting the differential diagnosis of brain metastases from benign and low-grade gliomas and necrosis in irradiated patients ${ }^{[29]}$.

\section{TREATMENT}

The therapeutic approach to patients with brain metastasis is intended to relieve symptoms, such as headache, vomiting, and neurological focalization; the success of this largely depends on the presence of cerebral hypertension syndrome secondary to perilesional cerebral edema. Surgical management continues to be the standard of treatment, accompanied by radiotherapy using two different techniques: stereotactic radiosurgery (SRS) or whole-brain radiation therapy (WBRT) (or a combination of these) ${ }^{[30]}$.

\section{Management of brain edema}

The treatment of cerebral edema with systemic steroids such as dexamethasone has been practiced for more than five decades ${ }^{[31]}$. Its efficacy is well known in terms of the clinical improvement of symptoms associated with vasogenic edema through its reduction of capillary permeability and regulation of the elevation of tight endothelial junctions involved in the physiology of the blood-brain barrier by binding to glucocorticoid receptors $^{[32-34]}$.

In actuality, the pharmacological measures previously adopted for the management of edema, including osmotic diuretics such as mannitol and glycerol and loop diuretics such as furosemide, are no longer considered standard for the treatment of cerebral edema secondary to metastasis. Because osmotic diuretics cause a redistribution of fluid from the intracellular space to the extracellular space, they reach a plateau within a few hours, reducing their effectiveness ${ }^{[35]}$.

Even though there is not a defined dexamethasone scheme, the most utilized dose in patients with clinical cerebral edema is 4 to $8 \mathrm{mg} / \mathrm{day}^{[33]}$.

According to a recent joint update of the American Society of Clinical Oncology and the Society for NeuroOncology, they endorse a practice guideline regarding the role of steroids in brain tumors. The recommended dose depends on symptom severity with an initial dose of $8 \mathrm{mg}$ in mildly symptomatic patients and $16 \mathrm{mg}$ in severely symptomatic patients ${ }^{[36]}$.

\section{Management of seizures}

In patients who have not presented with seizures, the use of an antiepileptic agent in a prophylactic manner is currently not recommended, following a systematic review by Perry et $a l^{[37]}$. In that study, no significant differences were found in reduction of risk of convulsive episodes in patients who were recently diagnosed with brain metastases and had never presented with seizures, between administration of the drug and only observation. 
Table 2. RPA classes

\begin{tabular}{lccc}
\hline Prognostic factors & Class I & Class II & Class III \\
\hline Age (years) & $<65$ & Any & Any \\
Controlled primary tumor & Yes & Any & Any \\
KPS & $>70$ & $>70$ & $<70$ \\
Extracranial metastasis & No & Any & Any \\
Estimated survival (months) & 7.1 & 4.2 & 2.3 \\
\hline
\end{tabular}

Abbreviations: RPA: recursive partition analysis; KPS: Karnofsky performance score. *All patients not in class I or III.

By contrast, the use of anticonvulsants in patients with seizures, although it does not have an impact on overall survival, is useful for the relief and decrease of the number of convulsive episodes. The drugs used most often that have been proven to be effective and safe include levetiracetam, oxcarbazepine, and topiramate $e^{[38,39]}$.

\section{INITIAL ASSESSMENT}

A therapeutic approach must be preceded by an adequate assessment of the patient's condition, evaluating parameters such as functional status and extracranial disease so that the best available treatment may be offered, without compromising the oncological outcome or overtreating patients in whom poor survival may be anticipated and for whom support measures are more adequate.

\section{PROGNOSTIC SCALES IN BRAIN METASTASES}

At present, several useful prognostic scales are available for the clinical decision-making process, the first of which is recursive partition analysis (RPA by Gaspar et al. ${ }^{[40]}$ ), developed in 1997. This scale was formed by the Radiation Therapy Oncology Group (RTOG) clinical trials that defined three prognostic classes according to four prognostic factors: performance according to the Karnofsky performance scale (KPS), control of the primary disease, presence or absence of extracerebral disease, and age greater or less than 65 years, dividing patients with brain metastases and their respective median survivals into three prognostic classes [Table 2].

Patients with a class III RPA are usually candidates for only supportive care, with local management performed either through surgery or radiotherapy for patients with classes I and $\mathrm{II}^{[40,41]}$.

The histological type of the primary tumor should also be considered an important prognostic factor; however, the previous scale does not take this into account. There is a more specific scale for primary disease that takes melanoma, gastrointestinal tumors, breast cell carcinoma, renal cell carcinoma and non-smallcell lung carcinoma into account. This scale is known as DS-GPA ${ }^{[2,43]}$.

The prognosis derived from these scales depends on various prognostic factors in relation to each histology, namely, age, KPS score, presence or absence of extracranial metastasis in the case of lung carcinoma, number of cerebral metastases in the case of lung carcinoma, melanoma, and renal cell carcinoma. Likewise, breast cancer, with its several molecular patterns that determine prognosis, is integrated into this scale [Table 3].

For more information on the estimation of global survival according to tumor type and characteristics, we recommend visiting a website made for this purpose: http://brainmetgpa.com/.

\section{SURGICAL MANAGEMENT}

Surgery plays an important role in the management of brain metastases, enabling a definitive histologic diagnosis in patients with no previously known history of cancer, allowing clinicians to alleviate the symptoms of intracranial hypertension (thus providing immediate relief to patients), and serving as a 
Table 3. Disease-specific GPA

\begin{tabular}{|c|c|c|c|c|c|c|}
\hline \multicolumn{7}{|l|}{ Disease-specific GPA } \\
\hline \multirow{2}{*}{ Histology } & \multirow{2}{*}{ Prognostic factors } & \multicolumn{5}{|c|}{ Score } \\
\hline & & 0 & 0.5 & & 1 & \\
\hline \multirow[t]{6}{*}{ NSCLC/SCLC } & AGE & $>60$ & $50-60$ & & $<50$ & \\
\hline & KPS & $<70$ & $70-80$ & & $90-100$ & \\
\hline & ECM & YES & & & NO & \\
\hline & \#BM & $>3$ & $2-3$ & & 1 & \\
\hline & & \multicolumn{5}{|c|}{ Score } \\
\hline & & 0 & 1 & & 2 & \\
\hline \multirow[t]{4}{*}{ MELANOMA/RCC } & KPS & $<70$ & $70-80$ & & $90-100$ & \\
\hline & \#BM & $>3$ & $2-3$ & & 1 & \\
\hline & & \multicolumn{5}{|c|}{ Score } \\
\hline & & 0 & 0.5 & 1 & 1.5 & 2 \\
\hline \multirow[t]{5}{*}{ BREAST } & KPS & $<60$ & 60 & $70-80$ & $90-100$ & \\
\hline & ER/PR/HER2 & Triple negative & & $\operatorname{ER} / \operatorname{PR}(+), \operatorname{HER} 2(-)$ & ER/PR $(-)$, HER2 $(+)$ & Triple positive \\
\hline & AGE & $>70$ & $<70$ & & & \\
\hline & & \multicolumn{5}{|c|}{ Score } \\
\hline & & 0 & 1 & 2 & 3 & 4 \\
\hline GASTROINTESTINAL & KPS & $<70$ & 70 & 80 & 90 & 100 \\
\hline
\end{tabular}

Abbreviations: GPA: graded prognostic index; NSCLC: non-small-cell lung carcinoma; SCLC: small-cell lung carcinoma; RCC: renal cell carcinoma; KPS: Karnofsky performance score; ECM: extracranial metastasis; \#BM: number of brain metastases; ER: estrogen receptor; PR: progesterone receptor; HER2: human epidermal growth factor receptor 2

primary therapeutic approach. However, in recent years it has been displaced by advanced radiotherapy techniques such as SRS.

The American Society for Radiation Oncology recommends surgical resection in patients with an expected survival of at least 3 months, lesions larger than $3-4 \mathrm{~cm}$, and who are amenable to safe, complete resection followed by WBRT or SRS to the cavity ${ }^{[44]}$.

\section{Whole-brain radiotherapy}

WBRT has been considered a mainstay treatment for brain metastases since the publication of Chao et al. ${ }^{[45]}$, who proposed irradiating the whole brain through two opposed lateral fields with the inferior margin of each field lying along the line running from the supraorbital ridge through the external auditory meatus to the foramen magnum; the other margins of the rectangular field project $2 \mathrm{~cm}$ beyond the forehead, vertex, and occiput onto bolus bags that surround the head. This was the first WBRT technique described using $250 \mathrm{kv}$ X-rays in 38 patients with brain metastasis. The authors reported that $63 \%$ of the enrolled patients demonstrated reduced symptoms associated with brain metastasis, with a relief duration of 3-4 months ${ }^{[45]}$.

\section{Dose and fractionation for WBRT}

Dose and fractionation schemes are based, not on the radiation sensitivity of the primary tumor, but rather on the tolerance of healthy brain tissue as described in the QUANTEC report from 2010 (maximum dose [Dmax] of 60 Gy with an estimated rate of symptomatic brain necrosis of $3 \%)^{[46]}$. Taking this into account and with a biologically equivalent dose (BED) with an $\alpha / \beta$ ratio of 3 for a normal brain, we cite the most used radiation schemes with their BED in Table 4.

Because the primary objective of this type of treatment is the palliation of symptoms, the most common prescription is $30 \mathrm{~Gy}$ in 10 fractions. This is based on the results of the first two randomized trials conducted by the RTOG, in which they compared four different radiation schemes including 3000 rad delivered in 2 weeks and 2000 rad in 1 week, and reported no differences in survival, time to progression, and symptom relief [Table 5 $]^{[47]}$. 
Table 4. Most used radiation therapy schemes for WBRT

\begin{tabular}{lc}
\hline Dose and fractionation & BED (Gy) \\
\hline $30 \mathrm{~Gy} / 2$ weeks & 60 \\
$20 \mathrm{~Gy} / 1$ week & 46.67 \\
$37.5 \mathrm{~Gy} / 3$ weeks & 68.75 \\
$40 \mathrm{~Gy} / 4$ weeks & 66.67 \\
\hline
\end{tabular}

Abbreviations: BED: Biologically equivalent dose. Gy: Gray

Table 5. Former RTOG dose and fractionation protocols for brain metastases

\begin{tabular}{|c|c|c|c|}
\hline \multicolumn{4}{|c|}{ RTOG brain metastasis protocols } \\
\hline First study & & & \\
\hline Scheme & \# patients & Scheme & \# patients \\
\hline $3000 \mathrm{rad} / 2$ weeks & 233 & $2000 \mathrm{rad} / 2$ weeks & 447 \\
\hline $3000 \mathrm{rad} / 3$ weeks & 217 & & \\
\hline $4000 \mathrm{rad} / 3$ weeks & 233 & $3000 \mathrm{rad} / 2$ weeks & 228 \\
\hline $4000 \mathrm{rad} / 4$ weeks & 227 & $4000 \mathrm{rad} / 3$ weeks & 227 \\
\hline
\end{tabular}

Considerations that can be taken into account as the physician decides on one fractionation scheme over another are the patient's performance status, estimated survival, and histology of the primary tumor because choriocarcinoma, melanoma, and renal cell carcinoma, among other types, present a higher risk of bleeding ${ }^{[43,48,49]}$.

The shorter-course fractionation of 20 Gy in 1 week is preferable for most patients with poor performance status, to avoid unnecessary treatment time, as it has demonstrated similar survival benefits as longer treatment schemes ${ }^{[50]}$. However, other fractionation schemes such as $37.5 \mathrm{~Gy}$ in 3 weeks is recommended in patients who have received a stereotactic radiosurgery boost with one metastatic lesion and should be considered in patients with one to three lesions ${ }^{[51]}$.

WBRT, unlike SRS, is associated with lower intracranial relapse, but when the whole brain is irradiated with this technique, it may also lead to greater cognitive deterioration (reflected as short-term memory loss), especially in patients with a longer life expectancy (> 6 months). In Aoyama et al. ${ }^{[52]}$, global survival did not significantly differ between treatment techniques ( $8.0 \mathrm{vs} .7 .5$ months) but there was a difference in the presentation of new metastases $(63.7 \%$ vs. $41.5 \%)$ WBRT as shown Figure 5.

\section{Role of Radiosurgery in the treatment of brain metastases}

SRS-based treatment began in 1951, with its implementation by Lars Leksell. It uses multiple rays of radiation, which converge three-dimensionally on a localized objective, either static or mobile, giving a high dose to a unique fraction with a high fall-off. This minimizes the damage to the adjacent tissue $e^{[53]}$.

Luckily, more than half of brain metastasis patients present with three or fewer lesions at diagnosis. It has been demonstrated that both surgical treatment and SRS lead to longer overall survival in these patients, especially for lone lesions smaller than $30 \mathrm{~mm}$, where SRS has an overall survival comparable to microsurgery. However, it is important to take into account that although brain metastases tend not to invade more than a few millimeters of adjacent tissue, local recurrences are common after resection, meaning that adjuvant treatment with radiotherapy after surgery is imperative ${ }^{[54]}$. To reduce cognitive impairment in such patients, the use of SRS has grown in use as an alternative to WBRT in the first 6 weeks following surgery, with the goal being to maintain local control in surgery and preserve neurocognitive functions without lowering quality of life ${ }^{[52]}$.

Clinical presentation with a single metastasis appears in only $10 \%$ to $20 \%$ of patients, where treatment with SRS following surgery improves both local recurrence rates and death due to neurological causes ${ }^{[5,56]}$. 



Figure 5. Whole-brain radiation therapy treatment plan using a 3D conformal technique with two opposite lateral fields

One of the first aleatorized studies employed SRS as a boost in patients with one to three brain metastases that had previously received treatment with WBRT, showing an improvement in functional status in all patients and an improvement in overall survival in patients with a single metastasis ${ }^{[2,57]}$.

The retrospective series published by Wang et al. ${ }^{[58]}$ in 2015 analyzed patients with brain metastases, comparing GammaKnife SRS alone, GammaKnife SRS with WBRT, surgery and SRS (as an adjuvant treatment to the surgical cavity), and a triple modality (surgery, SRS, and WBRT). For patients with a single metastasis and those with multiple lesions, the triple modality treatment was found to have greater positive effects on median survival than GammaKnife SRS alone. That study was not a prospective trial, and it also found better results for bimodal treatment than for GammaKnife surgery alone (as opposed to previous clinical trials). The authors concluded that WBRT is a good alternative as a rescue treatment for patients who had previously received SRS ${ }^{[58,59]}$.

SRS has broadened the terrain of the primary treatment of brain metastasis, especially in patients with good functional status and in those who have one to three metastases at diagnosis with limited extracranial disease $^{[58]}$ [As shown in Figures 6 and 7]. Therefore, it is important to note that better global control of metastasis can be obtained with WBRT and SRS, which have an impact on local control and overall survival $^{[51]}$. Patients whose disease is more limited in extent (up to four metastases for SRS protocols) may be treated with WBRT and SRS or SRS alone without improvement in their overall quality of life. A worsening of cognitive performance is found in patients treated with WBRT plus SRS, which affects their quality of life and ultimately their overall survival. These results were found by the trials conducted by Brown et al. ${ }^{[60]}$ and Chang et al. ${ }^{[6]}$. ASTRO and NCCN guidelines agree in recommending management with SRS in patients with a limited number of brain metastases.

The utility of SRS for patients with five or more brain metastases is unclear. The only prospective study that has evaluated patients with these characteristics was conducted by Yamamoto et al. ${ }^{[62]}$, who assessed 208 patients with 5 to 10 metastases, 531 patients with 2 to 4 metastases, and 455 patients with a single metastasis, with a maximum lesion diameter of $3 \mathrm{~cm}$. The most important result was that the number of brain metastases did not affect overall survival, while the volume of intracranial tumors ranged from 0.02 to $13.9 \mathrm{cc}$, and the average survival for patients with 5 to 10 metastases was 10.8 months. Deaths from neurological causes did not exceed $10 \%$ and there were no significant differences among groups. Finally, it was concluded that the progression of systemic disease was the main cause of death, the initial number of 




Figure 6. Treatment-planning dose for SRS of a single lesion, with the dose distribution for one target prescribing 20 Gy $95 \%$ with the following specification: isodose lines: red $20 \mathrm{~Gy}$, yellow $18 \mathrm{~Gy}$, blue $16 \mathrm{~Gy}$, brown $12 \mathrm{~Gy}$, pink $6 \mathrm{~Gy}$. Software: Eclipse External Beam Planning system version 15.5 HD MLC

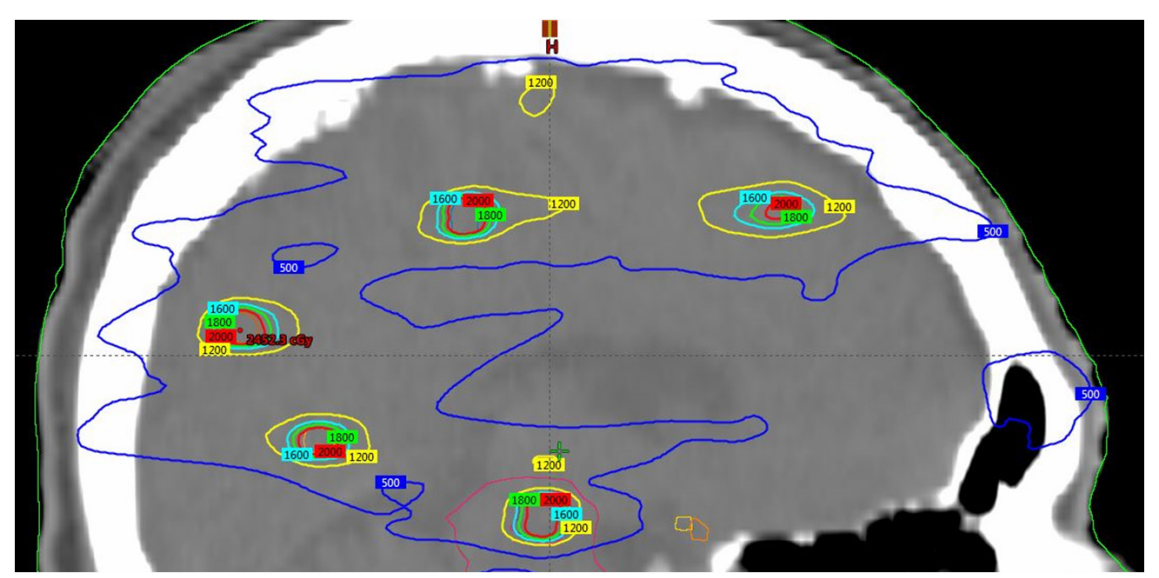

Figure 7. Multiple-target planning showing the dose distribution for multiple targets, prescribing $20 \mathrm{~Gy} 95 \%$ to each of them with the following specification: isodose lines: red $20 \mathrm{~Gy}$, green $18 \mathrm{~Gy}$, light blue $16 \mathrm{~Gy}$, yellow $12 \mathrm{~Gy}$, dark blue $6 \mathrm{~Gy}$. Software: Eclipse External Beam Planning system version 15.5 HD MLC

metastases did not impact local control, and the rate of distant metastasis failure was lower in patients with a single metastasis, although this advantage seemed to be lost for those with two or more metastases ${ }^{[62]}$.

Because $50 \%$ of patients with brain metastases will present with new lesions, it is necessary to continue surveillance using serial MRI when only SRS is used, with the intention of identifying the progression of the disease and enabling early management to limit neurological deficits. Success in the control of new distant metastases varies from $22 \%$ to $90 \%$, with different results reported by different studies. Many such studies have reported a correlation between the initial number of metastases and the progression of disease (according to Chang et al. ${ }^{[61]}$, the presence of 15 or more metastases is a risk factor). In addition to age, KPS, histology, and RPA, the total irradiated volume is also likely to impact overall survival, however, neither the number of metastases nor the total volume of treatment is yet considered a criterion for the selection of patients for treatment with SRS ${ }^{[51]}$.

Brown's and Chang's trial ${ }^{[60,61]}$ indicated that treatment with WBRT affects cognitive function in a significant way in all analyzed aspects, and opinions on the management of brain metastases converge on the use of SRS, even for multiple brain metastases, to avoid cognitive deficit ${ }^{[55]}$. 


\section{SRS TREATMENT DOSE}

As described in the protocol RTOG 9005, treatment dose is inversely proportional to metastatic lesion size. The suggested dose is $24 \mathrm{~Gy}$ for tumors smaller than $20 \mathrm{~mm}, 18 \mathrm{~Gy}$ for tumors from 21 to $30 \mathrm{~mm}$, and $15 \mathrm{~Gy}$ for tumors from 31 to $40 \mathrm{~mm}^{[63]}$. It is not known whether the dose used for lesions smaller than 20 $\mathrm{mm}$ can be safely incremented above $27 \mathrm{~Gy}$; hence, the general consensus still recommends a 24 Gy dose. However, because the organs at risk are so near, a single-dose treatment modality is associated with higher rates of toxicity, meaning that hypofractionated treatment plans are more appropriate for local control with acceptable toxicity ${ }^{[64]}$.

In 2014, Minniti published results of a study of hypofractionated SRS in which lesions under $20 \mathrm{~mm}$ received $36 \mathrm{~Gy}$ in three fractions, and lesions larger than $20 \mathrm{~mm}$ received $27 \mathrm{~Gy}$ in three fractions, resulting in 2 years of local control and an overall survival rate of $72 \%$ and $25 \%$, respectively ${ }^{[6]}$. In 2016 , Navarria published the results of a similar study, administering a dose of $27 \mathrm{~Gy}$ in three fractions to lesions of 21 to $30 \mathrm{~mm}$ and a dose of $32 \mathrm{~Gy}$ in four fractions to lesions of 31 to $50 \mathrm{~mm}$. The technique resulted in local control and an overall survival at 2 years of $96 \%$ and $33 \%$, respectively ${ }^{[6]]}$.

\section{CONTOURING OF BRAIN METASTASES}

Before a treatment plan is complete, an MRI with gadolinium contrast should be performed for fusion with axial CT. GTV is defined as gadolinium enhancement, identified in MRI with a $1 \mathrm{~mm}$ margin. A 1-3 mm margin is used for GTV in a geometrical form to obtain the total treatment volume (planning target volume) ${ }^{[63]}$.

\section{SRS boost after WBRT}

Several randomized trials that employed SRS as a boost after WBRT in newly detected patients with one to three surgically unresectable brain metastases have reported no added toxicity and improved performance in all patients and also an important benefit in the overall survival of patients with a single metastasis ${ }^{[1,52,57]}$. In addition, a recent review of five studies involving 2,728 patients reported a survival benefit in all patients who received a combined modality based on prognostic criteria (RPA or DS-GPA) regardless of the number of brain metastases ${ }^{[67]}$.

\section{Cavity radiation therapy}

A panel of international experts has recommended the following to contour CTV in SRS for patients with completely resected brain metastases. CTV must include the entire surgical cavity showing enhancement of contrast, using axial images in the T1 sequence of brain MRI with gadolinium contrast, excluding edema as determined by MRI. CTV must include the surgical path shown in the MRI or CT. If the presurgical tumor is in contact with the dura mater, CTV must include a 5-10 mm margin, until overlap with bone structures is achieved. If the presurgical tumor is not in contact with the dura mater, the CTV must include a 1-5 mm margin, until contact with bone structures is achieved. If a presurgical tumor is in contact with the venous sinus, CTV must include a 1-5 mm margin along the sinus ${ }^{[68]}$.

\section{Late toxicity implications: radiation cognitive syndrome}

Radiation cognitive syndrome is a poorly understood entity, and currently there is no validated long-term treatment or prevention; 50\%-90\% of patients who receive WBRT exhibit disabling cognitive function including declines in learning capability, processing speed, memory, and attention. This undermines the patient's overall quality of life. Radiation is associated with inflammation, gliosis, demyelination, vascular abnormalities, and necrosis, which in turn may lead to such cognitive issues ${ }^{[69]}$.

Cognitive deterioration is usually reported 6 months to 1 year after WBRT, but may be seen as early as 1 month after treatment. In addition, cognitive decline tends to be progressive and irreversible. Many 
radiation and pharmacologic approaches to address this syndrome are being studied, and while further data are needed for any one medical solution to become the standard of care, the results generated to date suggest a positive outlook for future treatment options ${ }^{[70]}$.

As new radiotherapy techniques have appeared, treatment plans have been developed that, in addition to tumor control, aim to reduce cognitive deterioration through the preservation of the hippocampus. Indeed, the RTOG 0933 trial $^{[71]}$ demonstrated the preservation of cognitive function by reducing the dose to the hippocampus to not more than 9 Gy at $100 \%$ of the volume and a maximal hippocampal dose of 16 Gy. This was achieved by manually contouring the hippocampus on a fused MRI-CT image set and expanding by $5 \mathrm{~mm}$ to generate "hippocampal avoidance regions." The mean relative cognitive decline from baseline to 4 months was 7.0\%, significantly lower than in controls. The study demonstrated that conformal avoidance of the hippocampus during WBRT was associated with preservation of memory and quality of life. Nonetheless, this procedure poses a risk of generating new brain metastases within the avoidance regions. However, this risk is not fully quantified and further data are needed to validate this technique within a phase III setting.

\section{CONCLUSION}

Although brain metastasis is the most common malignant intracranial tumor, it is closely linked to unfavorable outcomes. Its incidence has increased dramatically, due to a greater number of newly diagnosed cancer patients and the broader therapeutic options available today, which have led to better disease control and longer overall survival. The majority of patients are not candidates for surgical resection, so radiotherapy remains the standard of care. The possibility of a cure for an oligometastatic disease has been gaining increasing attention in recent years. The management of these patients has changed immeasurably over the past few decades: not many years ago, the prognosis and survival of such patients was for a short life expectancy, with poor disease control. At present, there are several treatment options available. The choice among these modalities depends on several factors, such as the functional state of the patient and the availability of equipment and treatment techniques at the given medical center. Before the 1990 s there was no GPA prognostic scale, much less an RPA, which are quite useful for decision making.

To date, no prospective studies have evaluated the use of SRS relative to WBRT for patients with more than four brain metastases. However, the current tendency in several hospitals around the world is to avoid WBRT, due to the toxicity and neurological deterioration attendant on that treatment, especially in developed countries. Consequently, there has been a shift to highly sophisticated techniques, such as SRS. A randomized phase III study is currently running at The Odette Cancer Center and the Princess Margaret Cancer Center (University of Toronto) in patients with 5 to 20 cerebral metastases who are receiving treatment with SRS without WBRT versus SRS plus WBRT, with the primary outcome being to compare neurocognitive decline between the approaches, as this is a common late side effect in patients receiving radiotherapy.

\section{DECLARATIONS}

\section{Authors' contributions}

All the authors contributed in an equitable way in the conception, bibliographic search and writing of this review article.

\section{Availability of data and materials}

All figures and tables are provided by authors and are available upon request to the corresponding author.

\section{Financial support and sponsorship}

None. 


\section{Conflicts of interest}

All authors declared that there are no conflicts of interest.

\section{Ethical approval and consent to participate}

Not applicable.

\section{Consent for publication}

Not applicable.

\section{Copyright}

(c) The Author(s) 2019.

\section{REFERENCES}

1. Tabouret E, Bauchet L, Carpentier A. Épidémiologie des métastases cérébrales et tropisme cérébral. Bull Cancer 2013;100:57-62.

2. Schouten LJ, Rutten J, Huveneers HA, Twijnstra A. Incidence of brain metastases in a cohort of patients with carcinoma of the breast, colon, kidney, and lung and melanoma. Cancer 2002;94:2698-705.

3. Fidler IJ. The biology of brain metastasis: challenges for therapy. Cancer 2015;21:284-93.

4. Conrad CA. Chemotherapy for metastatic tumors to the central nervous system. Curr Oncol Rep 2001;3:490-4.

5. Guõmundsson KR. A survey of tumours of the central nervous system in Iceland during the 10-year period 1954-1963. Acta Neurologica Scandinavica 1970;46:538-52.

6. Percy AK, Elveback LR, Okazaki H, Kurland LT. Neoplasms of the central nervous system. Epidemiologic considerations. Neurol 1972;22:40e8.

7. Barnholtz-Sloan JS, Sloan AE, Davis FG, Vigneau FD, Lai P, et al. Incidence proportions of brain metastases in patients diagnosed (1973 to 2001) in the Metropolitan Detroit Cancer Surveillance System. J Clin Oncol 2004;22:2865-72.

8. Fox BD, Cheung VJ, Patel AJ, Suki D, Rao G. Epidemiology of metastatic brain tumors. Neurosurg Clin N Am 2011;22:1-6.

9. Schouten LJ, Rutten J, Huveneers HA, Twijnstra A. Incidence of brain metastases in a cohort of patients with carcinoma of the breast, colon, kidney, and lung and melanoma. Cancer 2002;94:2698-705.

10. Suki D. The epidemiology of brain metastasis. In: Sawaya R, editor. Intracranial metastases: current management strategies. Malden (MA): Blackwell; 2004. p. 20.

11. Posner JB. Brain metastases: 1995. A brief review. J Neurooncol 1996;27:287-93.

12. Franchino F, Rudà R, Soffietti R. Mechanisms and therapy for cancer metastasis to the brain. Front Oncol 2018;8:161.

13. Cooper JB, Ronecker JS, Tobias ME, Mohan AL, Hillard V, et al. Molecular sequence of events and signaling in brain metastases. Anticancer Res 2018;38:1859-77.

14. Svokos KA, Salhia B, Toms SA. Molecular biology of brain metastasis. Int J Mol Sci 2014;15:9519-30.

15. Wilhelm I, Molnár J, Fazakas C, Haskó J, Krizbai IA. Role of the blood-brain barrier in the formation of brain metastases. Int J Mol Sci 2013;14:1383-411.

16. Winkler F. The brain metastatic niche. J Mol Med (Berl) 2015;93:1213-20.

17. Hugen N, Van de Velde CJH, De Wilt JHW, Nagtegaal ID. Metastatic pattern in colorectal cancer is strongly influenced by histological subtype. Ann Oncol 2014;25:651-7.

18. Beasley KD, Toms SA. The molecular pathobiology of metastasis to the brain: a review. Neurosurg Clin N Am 2011;22:7-14.

19. Schellinger PD, Meinck HM, Thron A. Diagnostic accuracy of MRI compared to CC in patients with brain metastases. J Neurooncol 1999;44:275-81.

20. Kramer RA, Janetos GP, Perlstein G. An approach to contrast enhancement in computed tomography of the brain. Radiology 1975;116:641-7.

21. Ferrigno D, Buccheri G. Cranial computed tomography as a part of the initial staging procedures for patients with non-small-cell lung cancer. Chest 1994;106:1025-9.

22. Sze G, Milano E, Johnson C, Heier L. Detection of brain metastases: comparison of contrast-enhanced MR with unenhanced MR and enhanced CT. Am J Neuroradiol 1990;11:785-91.

23. Hakyemez B, Erdogan C, Gokalp G, Dusak A, Parlak M. Solitary metastases and high-grade gliomas: radiological differentiation by morphometric analysis and perfusion-weighted MRI. Clin Radiol 2010;65:15-20.

24. Ishimaru H, Morikawa M, Iwanaga S, et al. Differentiation between high-grade glioma and metastatic brain tumor using single-voxel proton MR spectroscopy. Eur Radiol 2001;11:1784-91.

25. Chiang IC, Kuo YT, Lu CY, Yeung KW, Lin WC, et al. Distinction between high-grade gliomas and solitary metastases using peritumoral 3-T magnetic resonance spectroscopy, diffusion, and perfusion imagings. Neuroradiol 2004;46:619-27.

26. Chuang MT, Liu YS, Tsai YS, Chen YC, Wang CK. Differentiating radiation-induced necrosis from recurrent brain tumor using MR perfusion and spectroscopy: a meta-analysis. PLoS One 2016;11:e141438.

27. Wang Q, Zhang J, Xu W, Chen X, Zhang J, et al. Role of magnetic resonance spectroscopy to differentiate high-grade gliomas from 
metastases. Tumour Biol 2017;39:1010428317710030.

28. Usinskiene J, Ulyte A, Bjørnerud A, Venius J, Katsaros VK, et al. Optimal differentiation of high- and low-grade glioma and metastasis: a meta-analysis of perfusion, diffusion, and spectroscopy metrics. Neuroradiology 2016;58:339-50.

29. Fan G, Sun B, Wu Z, Guo Q, Guo Y. In vivo single-voxel proton MR spectroscopy in the differentiation of high-grade gliomas and solitary metastases. Clin Radiol 2004;59:77-85.

30. Al-Okaili RN, Krejza J, Wang S, Woo JH, Melhem ER. Advanced MR imaging techniques in the diagnosis of intraaxial brain tumors in adults. Radiographics 2006;26 Suppl 1: S173-89.

31. Horská A, Barker PB. Imaging of brain tumors: MR spectroscopy and metabolic imaging. Neuroimaging Clin N Am 2010;20:293-310.

32. Verma A, Kumar I, Verma N, Aggarwal P, Ojha R. Magnetic resonance spectroscopy: Revisiting the biochemical and molecular milieu of brain tumors. BBA Clin 2016;5:170-8.

33. Galicich JH, French LA, Melby JC. Use of dexamethasone in treatment of cerebral edema associated with brain tumors. Lancet 1961;81:46-53.

34. Ryken TC, McDermott M, Robinson PD, Ammirati M, Andrews DW, et al. The role of steroids in the management of brain metastases: a systematic review and evidence-based clinical practice guideline. J Neurooncol 2010;96:103-14.

35. Forster C, Silwedel C, Golenhofen N, Burek M, Kietz S, et al. Occludin as direct target for glucocorticoid-induced improvement of blood-brain barrier properties in a murine in vitro system. J Physiol 2005;565:475-86.

36. Chang SM, Messersmith H, Ahluwalia M, Andrews D, Brastianos PK, et al. Anticonvulsant prophylaxis and steroid use in adults with metastatic brain tumors: ASCO and SNO endorsement of the congress of neurological surgeons guidelines. J Clin Oncol 2019;37:1130-5.

37. Perry J, Zinman L, Chambers A, Spithoff K, Lloyd N, et al. The use of prophylactic anticonvulsants in patients with brain tumours: a systematic review. Curr Oncol 2006;13:222-9.

38. Furuse M, Hirase T, Itoh M, Nagafuchi A, Yonemura S, et al. Occludin: a novel integral membrane protein localizing at tight junctions. J Cell Biol 1993;123:1777-88.

39. Maschio M, Dinapoli L, Gomellini S, Ferraresi V, Sperati F, et al. Antiepileptics in brain metastases: safety, efficacy and impact on life expectancy. J Neurooncol 2010;98:109-16.

40. Gaspar L, Scott C, Rotman M, Asbell S, Phillips T, et al. Recursive partitioning analysis (RPA) of prognostic factors in three radiation therapy oncology group (RTOG) brain metastases trials. Int J Radiat Oncol Biol Phys 1997;37:745-51.

41. Gaspar LE, Scott C, Murray K, Curran W. Validation of the RTOG recursive partitioning analysis (RPA) classification for brain metastases. Int J Radiat Oncol Biol Phys 2000;47:1001-6.

42. Sperduto PW, Kased N, Roberge D, Xu Z, Shanley R, et al. Summary report on the graded prognostic assessment: an accurate and facile diagnosis-specific tool to estimate survival for patients with brain metastases. J Clin Oncol 2012;30:419-25.

43. Villà S, Weber DC, Moretones C, Mañes A, Combescure C, et al. Validation of the new graded prognostic assessment scale for brain metastases: a multicenter prospective study. Radiat Oncol 2011;6:23.

44. Tsao MN, Rades D, Wirth A, Lo SS, Danielson BL, et al. Radiotherapeutic and surgical management for newly diagnosed brain metastases: an American society for radiation oncology evidence based guideline. Prac Radiat Oncol 2012;2:210-25.

45. Chao JH, Phillips R, Nickson JJ. Roentgen-ray therapy of cerebral metastases. Cancer 1954;7:682-9.

46. Marks LB, Yorke ED, Jackson A, Ten Haken RK, Constine LS, et al. Use of normal tissue complication probability models in the clinic. Int J Radiat Oncol Biol Phys 2010;76:S10-9.

47. Borgelt B, Gelber R, Kramer S, Brady LW, Chang CH, et al. The palliation of brain metastases: Final results of the first two studies by the radiation therapy oncology group. Int J Radiat Oncol Biol Phys 1980;6:1-9.

48. Gaspar LE, Scott C, Murray K, Curran W. Validation of the RTOG recursive partitioning analysis (RPA) classification for brain metastases. Int J Radiat Oncol Biol Phys 2000;47:1001-6.

49. Sperduto PW, Kased N, Roberge D, Xu Z, Shanley R, et al. Summary report on the graded prognostic assessment: an accurate and facile diagnosis-specific tool to estimate survival for patients with brain metastases. J Clin Oncol 2012;30:419-25.

50. Rades D, Bohlen G, Dunst J, Lohynska R, Veninga T, et al. Comparison of short-course versus long-course whole-brain radiotherapy in the treatment of brain metastases. Strahlenther Onkol 2008;184:30-5.

51. Andrews DW, Scott CB, Sperduto PW, Flanders AE, Gaspar LE, et al. Whole brain radiation therapy with or without stereotactic radiosurgery boost for patients with one to three brain metastases: phase III results of the RTOG 9508 randomised trial. Lancet 2004;363:1665-72.

52. Aoyama H, Shirato H, Tago M, Nakagawa K, Toyoda T, et al. Stereotactic radiosurgery plus whole-brain radiation therapy vs. stereotactic radiosurgery alone for treatment of brain metastases: a randomized controlled trial. JAMA 2006;295:2483-91.

53. O’Beirn M, Benghiat H, Meade S, Heyes G, Sawlani V, et al. The expanding role of radiosurgery for brain metastases. Medicines (Basel) 2018;5:E90.

54. David Roberge, Parney I, Brown PD. Radiosurgery to the postoperative surgical cavity: who needs evidence? Int J Radiation Oncol Biol Phys 2012;83:486-93.

55. Flores BC, Patel AR, Timmerman RD, Barnett SL. From patchell to brown: an evidence-based evolution of the role of radiotherapy on the management of brain metastases. World Neurosurg 2016;85:10-4.

56. Patchell RA, Tibbs PA, Walsh JW, Dempsey RJ, Maruyama Y, et al. A randomized trial of surgery in the treatment of single metastases to the brain. N Engl J Med 1990;322:494-500.

57. Patchell RA, Tibbs PA, Regine WF, Dempsey RJ, Mohiuddin M, et al. Postoperative radiotherapy in the treatment of single metastases to the brain: a randomized trial. JAMA 1998;280:1485-9. 
58. Wang TJ, Saad S, Qureshi YH, Jani A, Isaacson SR, et al. Outcomes of gamma knife radiosurgery, bi-modality \& tri-modality treatment regimens for patients with one or multiple brain metastases: the Columbia University Medical Center experience. J Neurooncol 2015;122:399-408

59. Pirzkall A, Debus J, Lohr F, Fuss M, Rhein B, et al. Radiosurgery alone or in combination with whole-brain radiotherapy for brain metastases. J Clin Oncol 1998;16:3563-9.

60. Brown PD, Jaeckle K, Ballman KV, Farace E, Cerhan JH, et al. Effect of radiosurgery alone vs. radiosurgery with whole brain radiation therapy on cognitive function in patients with 1 to 3 brain metastases, JAMA 2016;316:401-9.

61. Chang EL, Wefel JS, Hess KR, Allen PK, Lang FF, et al. Neurocognition in patients with brain metastases treated with radiosurgery or radiosurgery plus whole-brain irradiation: a randomised controlled trial. Lancet Oncol 2009;10:1037-44.

62. Yamamoto M, Serizawa T, Shuto T, Akabane A, Higuchi Y, et al. Stereotactic radiosurgery for patients with multiple brain metastases (JLGK0901): a multi-institutional prospective observational study. Lancet Oncol 2014;15:387-95.

63. Shaw E, Scott C, Souhami L, Dinapoli R, Kline R, et al. Single dose radiosurgical treatment of recurrent previously irradiated primary brain tumors and brain metastases: final report of RTOG protocol 90-05. Int J Radiation Oncol Biol Phys 2000;47:291-8.

64. Riccardo Soffietti, Abacioglu U, Baumert B, Combs SE, Kinhult S, et al. Diagnosis and treatment of brain metastases from solid tumors: guidelines from the European Association of Neuro-Oncology (EANO). Neuro Oncol 2017;19:162-74.

65. Minniti G, D'Angelillo RM, Scaringi C, Trodella LE, Clarke E, et al. Fractionated stereotactic radiosurgery for patients with brain metastases. J Neuro-Oncol 2014;117:295-301.

66. Navarria P, Pessina F, Cozzi L, Ascolese AM1, De Rose F, et al. Hypo-fractionated stereotactic radiotherapy alone using volumetric modulated arc therapy for patients with single, large brain metastases unsuitable for surgical resection. Radiat Oncol 2016;11:76.

67. Khan M, Lin J, Liao G, Tian Y, Liang Y, et al. Whole brain radiation therapy plus stereotactic radiosurgery in the treatment of brain metastases leading to improved survival in patients with favorable prognostic factors. Front Oncol 2019;9:205.

68. Soliman H, Ruschin M, Angelov L, Brown PD, Chiang VLS, et al. Consensus contouring guidelines for post-operative completely resected cavity stereotactic radiosurgery (SRS) for brain metastases. Int J Radiat Oncol Biol Phys 2017;100:436-42.

69. Makale M, McDonald C, Hattangadi-Gluth J, Kesari S. Brain irradiation and long-term cognitive disability: Current concepts. Nat Rev Neurol 2017;13:52-64.

70. Wu PH, Coultrap S, Pinnix C, Davies KD, Tailor R, et al. Radiation induces acute alterations in neuronal function. PLoS One 2012;7:e37677.

71. Gondi V, Pugh SL, Tome WA, Caine C, Corn B, et al. Preservation of memory with conformal avoidance of the hippocampal neural stem-cell compartment during whole-brain radiotherapy for brain metastases (RTOG 0933): a phase II multi-institutional trial. J Clin Oncol 2014;32:3810-6. 